\title{
EDITORIAL
}

\section{JHG Young Scientist Award}

(c) The Author(s), under exclusive licence to The Japan Society of Human Genetics 2021

Journal of Human Genetics (2022) 67:69; https://doi.org/10.1038/ s10038-021-00994-y

JHG Young Scientist Award recognizes articles that have made significant contribution to the Journal of Human Genetics, thanks to their scientific excellence and impact in the field of human genetics.

We are pleased to announce the winners of JHG Young Scientist Award for the years 2020 and 2021, respectively, as below. We would like to express our warmest congratulations, and wish them all the best in the future.

\section{0 winners}

Dr. Wenjuan Chen

Department of Pharmacology, Emory University School of Medicine

Dr. Christine Shieh

David Geffen School of Medicine at University of California

for the article entitled

GRIN1 mutation associated with intellectual disability alters NMDA receptor trafficking and function

Journal of Human Genetics 2017;62:589-597

https://www.nature.com/articles/jhg201719

Dr. Satoko Miyatake

Department of Human Genetics, Graduate School of Medicine, Yokohama City University for the article entitled

ANKRD11 variants cause variable clinical features associated with KBG syndrome and Coffin-Siris-like syndrome

Journal of Human Genetics 2017;62:741-746

https://www.nature.com/articles/jhg201724

\section{1 winners}

Dr. Kazuhiro Iwama

Department of Human Genetics, Graduate School of Medicine, Yokohama City University

for the article entitled

A novel SLC9A1 mutation causes cerebellar ataxia

Journal of Human Genetics 2018;63:1049-1054

https://doi.org/10.1038/s10038-018-0488-x

Dr. Weiyu Li

Obstetrics and Gynecology Hospital, NHC Key Laboratory of Reproduction Regulation (Shanghai Institute of Planned Parenthood Research), School of Life Sciences, Fudan University

for the article entitled

Biallelic mutations of CFAP251 cause sperm flagellar defects and human male infertility

Journal of Human Genetics 2019;64:49-54

https://doi.org/10.1038/s10038-018-0520-1

Toshihiro Tanaka ${ }^{1 \times}$

${ }^{1}$ Editor-in-Chief, Journal of Human Genetics, Tokyo, Japan.

凶email: ttana.brc@tmd.ac.jp 